\title{
An Intercomparison of Counting Efficiency and the Performance of Two Whole-Body Counters According to the Type of Phantom
}

\author{
Minjung Pak, Jaeryong Yoo, Wi-Ho Ha*, Young-Woo Jin \\ Korea Institute of Radiological and Medical Sciences, Seoul, Korea
}

\section{Technical Paper}

Received February 29, 2016

Revision June 15, 2016

Accepted June 16, 2016

Corresponding author: $\mathrm{Wi}-\mathrm{Ho} \mathrm{Ha}$

Korea Institute of Radiological and

Medical Sciences, 75, Nowon-gil,

Nowon-gu, Seoul 01812, Korea

Tel: +82-2-3399-5961

Fax: +82-2-3399-5960

E-mail: lovin@kirams.re.kr

This is an Open-Access article distributed under the terms of the Creative Commons Attribution NonCommercial License (http://creativecommons.org/ licenses/by-nc/4.0) which permits unrestricted noncommercial use, distribution, and reproduction in any medium, provided the original work is properly cited.

Copyright $\odot$ 2016 The Korean Association for Radiation Protection

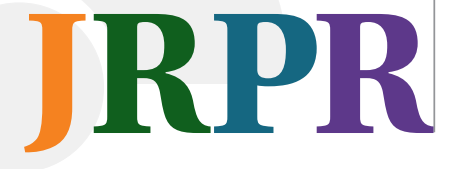

Background: Whole-body counters are widely used to evaluate internal contamination of the internal presence of gamma-emitting radionuclides. In internal dosimetry, it is a basic requirement that quality control procedures be applied to verify the reliability of the measured results. The implementation of intercomparison programs plays an important role in quality control, and the accuracy of the calibration and the reliability of the results should be verified through intercomparison. In this study, we evaluated the reliability of 2 whole-body counting systems using 2 calibration methods.

Materials and Methods: In this study, 2 whole-body counters were calibrated using a reference male bottle manikin absorption (BOMAB) phantom and a Radiation Management Corporation (RMC-II) phantom. The reliability of the whole-body counting systems was evaluated by performing an intercomparison with International Atomic Energy Agencyto assess counting efficiency according to the type of the phantom.

Results and Discussion: In the analysis of counting efficiency using the BOMAB phantom, the performance criteria of the counters were satisfied. The relative bias of activity for all radionuclides was -0.16 to 0.01 in the Fastscan and -0.01 to 0.03 in the Accuscan. However, when counting efficiency was analyzed using the RMC- II phantom, the relative bias of ${ }^{241} \mathrm{Am}$ activity was -0.49 in the Fastscan and 0.55 in the Accuscan, indicating that its performance criteria was not satisfactory.

Conclusion: The intercomparison process demonstrated the reliability of whole-body counting systems calibrated with a BOMAB phantom. However, when the RMC-II phantom was used, the accuracy of measurements decreased for low-energy nuclides. Therefore, it appears that the RMC-II phantom should only be used for efficiency calibration for high-energy nuclides. Moreover, a novel phantom capable of matching the efficiency of the BOMAB phantom in low-energy nuclides should be developed.

Keywords: Whole-body counter, Intercomparison, Counting efficiency, Phantom, Performance criteria, Quality control

\section{Introduction}

Assessing the intake of radionuclides is an important aspect of internal dosimetry. Bioassays used to measure intake of radionuclides can be divided into those that directly measure the in vivo amount of radioactive material and those that indirectly measure radioactive material through in vitro measurements of the radioactive materi- 
al released in excretions. Since in vivo bioassay is made from the outside of the body, only strongly penetrating gamma ray-emitting or X-ray emitting nuclides can be measured in this way. In vivo measurements are made either through direct monitoring, in which nuclide accumulation is measured in a particular organ, or through whole-body counting, in which nuclide accumulation distributed throughout the entire body is measured. Generally, whole-body counting is used to assess the internal contamination of most nuclides, except for those that accumulate in specific organs $[1,2]$.

Quality management systems should be put in place to ensure high reliability in measurements of the internal contamination of nuclides using whole-body counting. Stringent quality assurance provides confidence that the needs of consumers will be met, and this process generally necessitates the periodic evaluation of factors related to the fitness of the operational aspects of quality assurance [3]. Quality assurance ensures that the resulting measurements are in fact reliable [4]. Since measurements of internal contamination using whole-body counting are influenced by various factors that may contribute to uncertainty, it is all the more important to implement quality management systems involving intercomparison. Such quality management systems must be used to confirm the accuracy of the calibration of wholebody counters and the reliability of the measurements [4]. Therefore, laboratories that use whole-body counters have conducted intercomparison, which incorporate not only quality assurance of the measurements, but also have the goal of identifying the causes of errors and improving dosimetry techniques [5-10].

Accordingly, in this study, we performed an intercomparison of results reported by the International Atomic Energy Agency (IAEA) and the results obtained in our laboratory. Through the use of calibration phantoms, we evaluated the validity of whole-body counting systems according to the type of calibration phantom used.

\section{Materials and Methods}

\section{Whole-body counters}

We evaluated 2 whole-body counters that had different types of detectors and geometry: the Fastscan (Model 2250;
Canberra, Meriden, CT, USA) and the Accuscan (Model 2260; Canberra, Meriden, CT, USA).

The Fastscan is a stand up type whole-body counter, in which the subject stands encircled by a shield encompassing the detector, which rests parallel to the subject. The Fastscan contains $2 \mathrm{NaI}(\mathrm{T} 1)$ detectors with a dimension of $7.6 \times$ $12.7 \times 40.6 \mathrm{~cm}^{3}$ that are positioned serially at the top and bottom. The Fastscan was designed on the basis of anthropometric data; the detectors are positioned such that they are able to scan the entire body. The counter is able to monitor nuclides with energies between $300 \mathrm{keV}$ and $1.8 \mathrm{MeV}^{1)^{1,2)}}$

The Accuscan is a horizontal bed type whole-body counter in which the subject undergoes counting in a lying position rather than a standing position. Since the subject is in a relatively comfortable position, the length of the count can be extended and subjects with disabilities can be measured more easily. The Accuscan contains 2 high-purity Ge (HPGe) detectors. The HPGe detector has a relative efficiency of $25 \%$ to the $7.6 \times 7.6 \mathrm{~cm}^{2} \mathrm{NaI}(\mathrm{T} 1)$ detector. The scanning range of the detectors measures $2 \mathrm{~m}$ and fully encompasses the areas of nuclide deposition in the body, and this configuration reduces bias and inaccuracies in measurement arising from variations in body size or in the location of nuclide deposits. ${ }^{3)}$

Both types of whole-body counters are encased on all sides except the anterior face in 10-cm-thick, low-background, lead shielding. Counts from background radiation were eliminated by using specially-manufactured ${ }^{60} \mathrm{Co}$-free lead shielding.

\section{Efficiency calibration of whole-body counters}

The efficiency calibration of whole-body counters requires the use of a phantom that contains radioactive material with known certified values. Usually, the form and the mass attenuation coefficient of the calibration phantom are similar to the human body in order to reflect the counting efficiency that can be obtained using real subjects. In this study, we used an adult male bottle manikin absorption (BOMAB) phantom and a Radiation Management Corporation (RMCII) phantom to calibrate the counting efficiency of the wholebody counters. Further, we compared the counting efficiency of each phantom by photon energy relative to the standard counting efficiency of the BOMAB phantom. The calibrated

1) Canberra Inc. Model 2250 Fastscan high-throughput whole-body counter. 2002;1-3.

2) Bronson FL, Booth LF, Richards DC. A computerized, anthropometrically designed, high throughput, whole-body counter for the nuclear industry. Technical Memo of Canberra's Whole-body counter. 1998;6-8.

3) Canberra. Model 2260 Accuscan Horizontal bed whole-body counter. 2004;1-2. 
efficiency was expressed using a fourth-order polynomial function.

The BOMAB phantom is the standard calibration phantom for whole-body counting [11]. The energy source of this phantom is able to release over $200 \mathrm{keV}$ of photon energy, and it has been designed as a phantom for measuring isotopes homogenously distributed over the body [12, 13]. The BOMAB phantom was manufactured on the basis of a report of the International Commission on Radiological Protection (ICRP) that provided detailed anthropometric data [14]. The BOMAB phantom is composed of 10 cylinders corresponding to different body parts. Depending on the body part to which they correspond, the cylinders are either elliptical or circular. Elliptical cylinders are used for the head, chest, and gut, while circular cylinders are used for the calves, thighs, arms, and neck. Each cylinder is made of high-density polyethylene and the hollow space is filled with $0.1 \mathrm{M} \mathrm{HCl}$. Polyethylene is the preferred constituent material of phantoms because the mass attenuation coefficient of polyethylene remains within $10 \%$ of the mass attenuation coefficient of soft tissue across photon energies ranging from $200 \mathrm{keV}$ to $3 \mathrm{MeV}$, thereby closely resembling the conditions of soft tissue [12]. The certified reference material (CRM) used in the BOMAB phantom is a liquid solution containing 9 types of nuclides that emit gamma rays (Eckert \& Ziegler Analytics, Braunschweig, Germany). The range of energy emission of this CRM is $59.5-1,836.1 \mathrm{keV}$, and the relative expanded uncertainty of the activity is within $2.8 \%(\kappa=2)$. In order to simulate the homogeneous distribution of the isotope throughout the body, the CRM was homogenously distributed within the BOMAB phantom.

The RMC-II phantom was developed by Canberra (Meriden, CT, USA) to make phantoms more user-friendly and cost-effective. The counting efficiency of the American National Standards Institute (ANSI) N13.30 phantom was taken into account in its manufacture, and the RMC-II phantom was designed to be used for calibrating whole-body counters with a linear geometry [15]. The ANSI N13.30 phantoms, which are reflected in the RMC-II phantom, include the BOMAB phantom, the ANSI N44.3 thyroid phantom, and the Lawrence Livermore National Laboratory (LLNL) lung phantom [11]. The RMC-II phantom is manufactured so that 20 $\mathrm{mL}$ of a CRM can be inserted in any one of the 4 cavities, which are geometric imitations of the following anatomical structures (from top to bottom): the thyroid, the lungs, the whole body, and the gastrointestinal tract. The structure of the RMC-II phantom can be divided into the neck and body. The body is made from an acrylic board, the thickness of which is manufactured in a way that most accurately simulates how radiation sources are received by detectors. The neck is also made from an acrylic board and its cylindrical structure is manufactured according to the dimensions suggested in ANSI N44.3 [16]. When the RMC-II phantom is used to calibrate the counting efficiency, the height of the whole-body counter is leveled such that the floor of the phantom is at the level of the waist of the examiner (with a resulting height of $91 \mathrm{~cm}$ ) [15]. Thus, the phantom was placed at the appropriate height in the Fastscan and in the Accuscan to perform the efficiency calibration. The CRM used in the RMC-II phantom was sealed in a $20-\mathrm{mL}$ container; it contained eight types of nuclides in the form of a geltype mixed gamma source, and was manufactured by the Korea Research Institute of Standards and Science (KRISS). The rang e of the photon energy of the CRM is 59.5-1,836.1 $\mathrm{keV}$, which is similar to that of the CRM used in the BOMAB phantom, and the relative expanded uncertainty of activity is within $4.0 \%(\kappa=2)$. Of the ray portals of the RMC-II phantom, the portal most reflective of a homogeneously distributed isotope was used.

\section{Performance verification of the whole-body counters using the BOMAB phantom}

Performance test was conducted subsequent to the efficiency calibration. For the performance testing, we used a BOMAB phantom containing gamma ray-emitting nuclides distinct from CRM used for the efficiency calibration. The $B O M A B$ phantom used for verification testing had the same geometry as the male adult BOMAB phantom used for the efficiency calibration and included the following nuclides: ${ }^{241} \mathrm{Am},{ }^{134} \mathrm{Cs}$, ${ }^{137} \mathrm{Cs},{ }^{60} \mathrm{Co}$, and ${ }^{133} \mathrm{Ba}$. Of these, we used ${ }^{134} \mathrm{Cs}$ and ${ }^{133} \mathrm{Ba}$ to analyze the performance parameters, as these nuclides had energies different from those used during the calibration.

The whole-body verification counts for the Fastscan were collected for 240 seconds for the BOMAB phantom, whereas a duration of 1,800 seconds was used for the Accuscan. Each measurement was taken 5 times. These verification counting times were the same as those used for actual counting procedures. The measurements of both the Fastscan and Accuscan were analyzed using the counting efficiencies of the BOMAB phantom and the RMC-II phantom. Additionally, the measurements were evaluated in relation to the certified 
values of the verification $\mathrm{BOMAB}$ phantom in order to determine whether the counters were successfully calibrated. We assessed the measurements according to the International Organization for Standardization (ISO) 28218 criteria; specifically, we evaluated relative bias (Br) and repeatability $\left(S_{B r}\right)$, which can be used to estimate the accuracy and the precision of the measurement. The following performance criteria were applied: $-0.25<\mathrm{Br}<0.50$ and $S_{B r} \leqq 0.4$. We calculated $B_{r}$ and $S_{B r}$ using the equations presented below [17].

$$
B_{r i}=\frac{\left(A_{i}-A_{a i}\right)}{A_{a i}}
$$

In this equation, $B_{r i}$ denotes the relative bias of the $i^{\text {th }}$ measurement, Ai denotes the $i^{\text {th }}$ measurement of radioactivity, and $A_{a i}$ denotes the certified value of the radioactive sample. The relative bias $\left(B_{r}\right)$ is the average of the relative biases of the measurements and can be evaluated using the following equation:

$$
B_{r}=\sum_{i=1}^{n} \frac{B_{r i}}{n}
$$

Here, $n$ denotes the number of total measurements. Repeatability can be calculated using the following equation:

$$
S_{B r}=\sqrt{\frac{\sum_{i=1}^{n}\left(B_{r i}-B_{r}\right)^{2}}{(n-1)}}
$$

\section{Intercomparison of whole-body counters using sliced BOMAB phantoms}

The intercomparison necessitates transportation of the phantoms between laboratories. As they are designed to simulate the whole body, it is unsurprising that they have very large volumes, and their transportation is hazardous

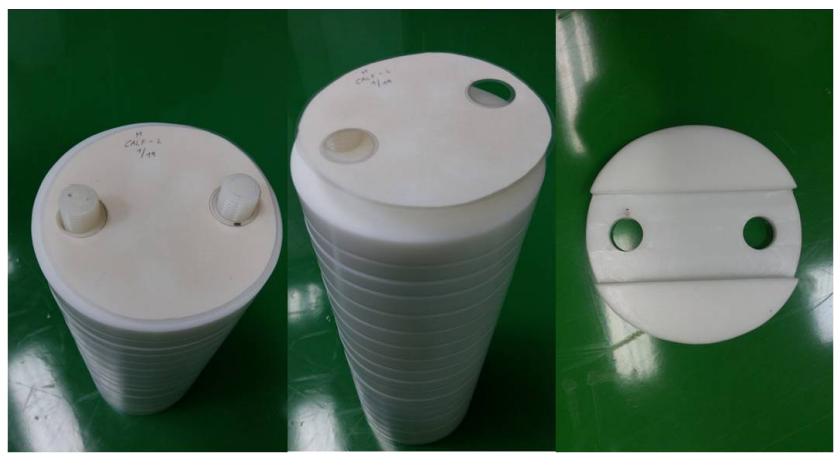

Fig. 1. The calf part of the International Atomic Energy Agency sliced bottle manikin absorption (BOMAB) phantom. due to potential radioactive leakage. IAEA sliced BOMAB phantoms, the radioactive material sealed in paper filters, was used in intercomparison in order to prevent incidents of radioactive leakage during delivery from the IAEA to the laboratory.

Previous intercomparison have shown that the agreement between the sliced BOMAB phantom and the conventional $\mathrm{BOMAB}$ phantom was $\pm 8 \%$ in whole-body counters of various geometries [18]. These findings support the fact that the sliced BOMAB phantom closely resembles the homogenous distribution of radioactive material in the body. The body of the sliced BOMAB phantom is divided into several slices, with the thickness of each slice either $1.9 \mathrm{~cm}$ or $1.27 \mathrm{~cm}$. The body is mainly made up of the thicker slices, and the thinner slices are integrated in between the larger slices to make the lengths of the phantom even [18]. As shown in Figure 1, each region of the sliced BOMAB phantom consists of 2 column, and the slices are connected to one another through these column. Between each slice, a surface source, which contains a homogeneous composite of the CRM, was inserted, and this conformation simulates how the radioactive material is homogeneously distributed across the body.

The counts were collected for 240 seconds for the Fastscan and for 1,800 seconds for the Accuscan; these durations are those used in actual procedures. Each measurement was repeated 5 times. Figure 2 shows how the the sliced ВОМАВ phantom was positioned in the Fastscan and in the Accuscan. The gamma spectrum of radionuclides that was measured with each whole-body counter was analyzed based on efficiency calibrations made using the BOMAB phantom and

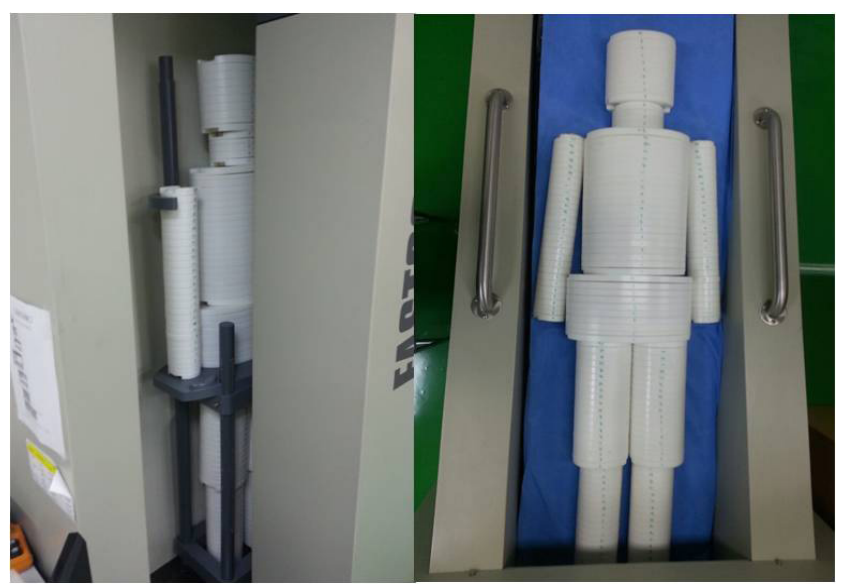

Fig. 2. The International Atomic Energy Agency sliced bottle manikin absorption (BOMAB) phantom positioned in the Fastscan (left) and Accuscan (right). 


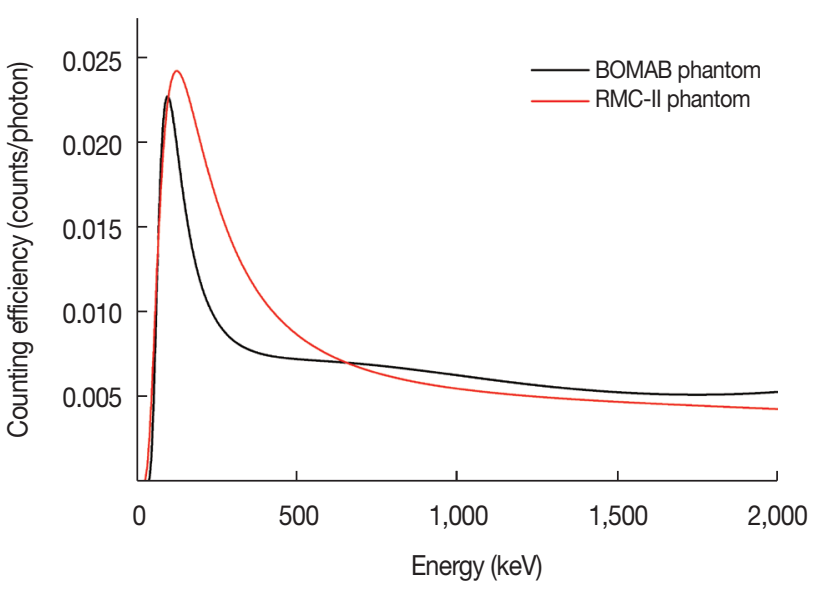

Fig. 3. Counting efficiency of the Fastscan calibrated using a bottle manikin absorption (BOMAB) phantom and a Radiation Management Corporation (RMC-II) phantom.

the RMC-II phantom. Additionally, we performed an intercomparison using the certified values of the sliced BOMAB phantom as the reference. The results of the intercomparison, comprising the assessment parameters and the ISO 28218 performance criteria, are illustrated in paragraph 2.3 .

\section{Results and Discussion}

\section{Results of the efficiency calibrations of the whole- body counters}

The counting efficiency of the Fastscan calibrated using the BOMAB phantom and the RMC-II phantom is presented in Figure 3. The disparity in counting efficiency between the phantoms at high photon energies (above $600 \mathrm{keV}$ ) was minimal (0\%-2\%). However, the disparity in counting efficiency between the phantoms at low photon energies (below $600 \mathrm{keV}$ ) was very large (-16\% to $47 \%)$. The disparity seen in counting efficiency at low photon energies is thought to stem from the fact that low photon energies tend to be more affected by the geometry of the counters [19].

The counting efficiency of the Accuscan calibrated using the BOMAB phantom and the RMC-II phantom is presented in Figure 4. We found that the disparity in counting efficiency between the phantoms was large across all photon energies for the Accuscan. We compared 2 distinct levels of photon energy (above and below $600 \mathrm{keV}$ ). We found that the disparity in counting efficiency between the 2 phantoms at low photon energies was between $-19 \%$ and $-33 \%$, defined relative to the BOMAB phantom counting efficiency. The disparity in the counting efficiency between phantoms at high

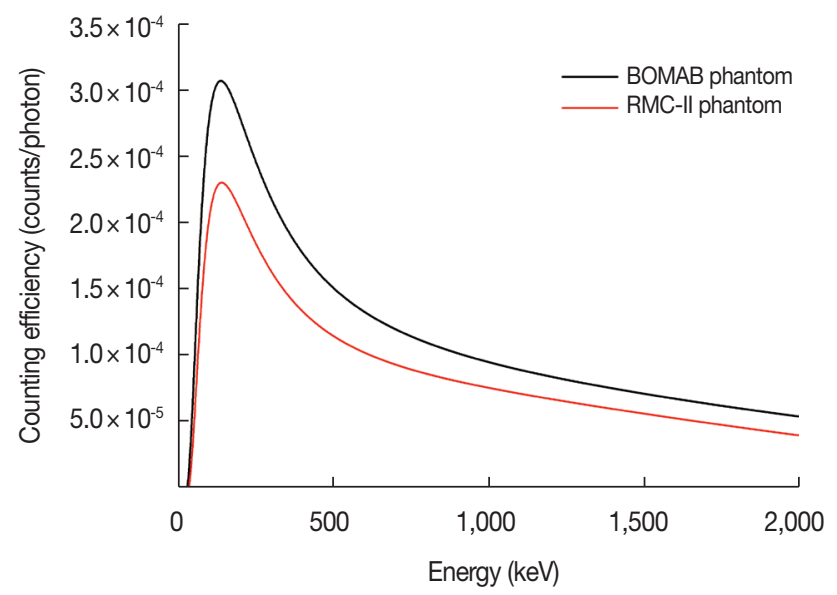

Fig. 4. Counting efficiency of the Accuscan calibrated using a bottle manikin absorption (BOMAB) phantom and Radiation Management Corporation (RMC-II) phantom.

photon energies ranged from $-7 \%$ to $-13 \%$; thus, the disparity was less prominent than that seen at low photon energies but larger than that observed in the Fastscan at equivalent energies. We believe that this striking disparity can be ascribed to the geometry of the Accuscan, which scans the body from the legs to the head. The Accuscan is able to scan the BOMAB phantom from the lower end to the upper end, but is able to scan only the region of the RMC-II phantom equivalent to the upper body of the subject; as the scanning area of the Accuscan fails to encompass the lower end of the RMC-II phantom, which in fact lies beyond the shielding of the detector.

For the RMC-II phantom to be a valid alternative to the conventional BOMAB phantom, the counting efficiency of the RMC-II phantom must be comparable to that of the conventional $B O M A B$ phantom, which is the standard phantom for calibration. However, we found that the counting efficiencies of the RMC-II phantom and the BOMAB phantom showed large discrepancies in the Fastscan at low photon energies (below $600 \mathrm{keV}$ ) and in the Accuscan counter across all photon energies. We suggest that further studies investigate this discrepancy.

\section{Performance testing of whole-body counters using a BOMAB phantom for validation}

A BOMAB phantom was used to calibrate the Fastscan and the Accuscan prior to analyzing them with the counting efficiency of either the RMC-II or the BOMAB phantom. The $S_{B r}$ and the $B_{r}$ of the measurements are summarized in Figure 5 . The bars with diagonal lines denote the performance 


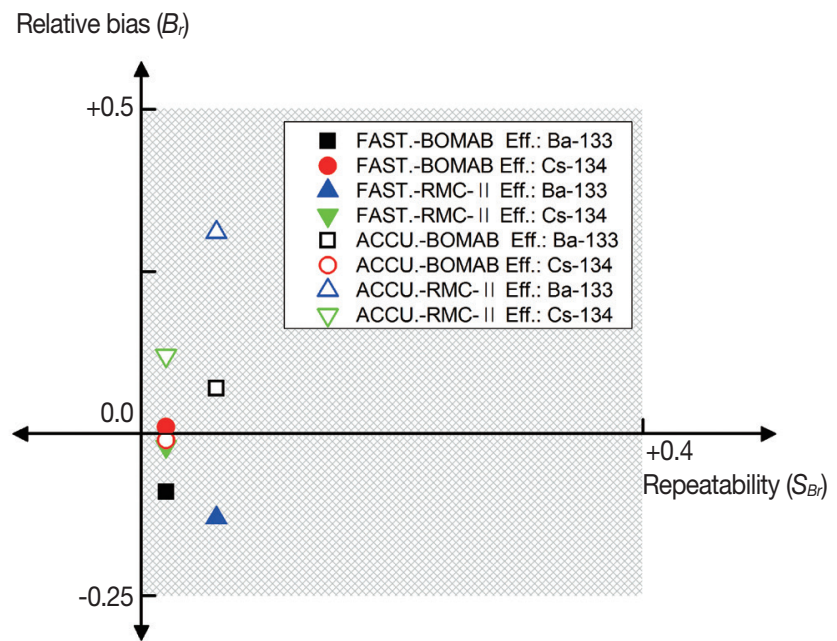

Fig. 5. Performance test results for the Fastscan and Accuscan.

criteria $\left(0.25<B_{r}<0.50\right.$ and $\left.S_{B r} \leqq 0.4\right)$ derived from bioassays as indicated in the ISO 28218 criteria. Measurements within these parameter ranges can be considered to indicate that the performance criteria were met.

When we analyzed the measurement results of the Fastscan using counting efficiency of the BOMAB phantom, we found that the $B_{r}$ of ${ }^{133} \mathrm{Ba}\left(356 \mathrm{keV}\right.$ ) was -0.09 and that the $B_{r}$ of ${ }^{134} \mathrm{Cs}(605 \mathrm{keV})$ was -0.06 . The $S_{B r}$ values of ${ }^{133} \mathrm{Ba}$ and ${ }^{134} \mathrm{Cs}$ were 0.02 . We likewise analyzed the results of the Fastscan using counting efficiency of the RMC-II phantom. The $B_{r}$ for

${ }^{133} \mathrm{Ba}$ was -0.13 , and the $B_{r}$ of ${ }^{134} \mathrm{Cs}$ was -0.02 . The $S_{B r}$ of ${ }^{133} \mathrm{Ba}$ was 0.06 , and that of ${ }^{134} \mathrm{Cs}$ was 0.02 . All analyzed results of Fastscan using 2 phantoms were within the acceptable performance criteria.

We analyzed the measurement results of the Accuscan using counting efficiency of the BOMAB phantom and the RMC-II phantom. For the BOMAB phantom, the $B_{r}$ of ${ }^{133} \mathrm{Ba}$ was 0.07 and the $B_{r}$ for ${ }^{134} \mathrm{Cs}$ was -0.01 . The $S_{B r}$ of ${ }^{133} \mathrm{Ba}$ was 0.06 and the $S_{B r}$ for ${ }^{134} \mathrm{Cs}$ was 0.02 . For the RMC-II phantom, the $B_{r}$ of ${ }^{133} \mathrm{Ba}$ was 0.31 and the $B_{r}$ of ${ }^{134} \mathrm{Cs}$ was 0.12 . The $S_{B r}$ of ${ }^{133} \mathrm{Ba}$ was 0.06 , and the $S_{B r}$ of ${ }^{134} \mathrm{Cs}$ was 0.02 . These findings show that the Accuscan satisfied the performance criteria regardless of the calibration phantom. However, using the counting efficiency of the RMC-II phantom led to a $B_{r}$ value of ${ }^{133} \mathrm{Ba}$ that was more than 4 times greater than that observed when the counting efficiency of the BOMAB phantom was used, and a $B_{r}$ value of ${ }^{134} \mathrm{Cs}$ that was more than 12 times greater. We therefore suggest that the assessment of radionuclides in the whole body with counting efficiency calibrated using the RMC-II phantom may impair the accuracy.

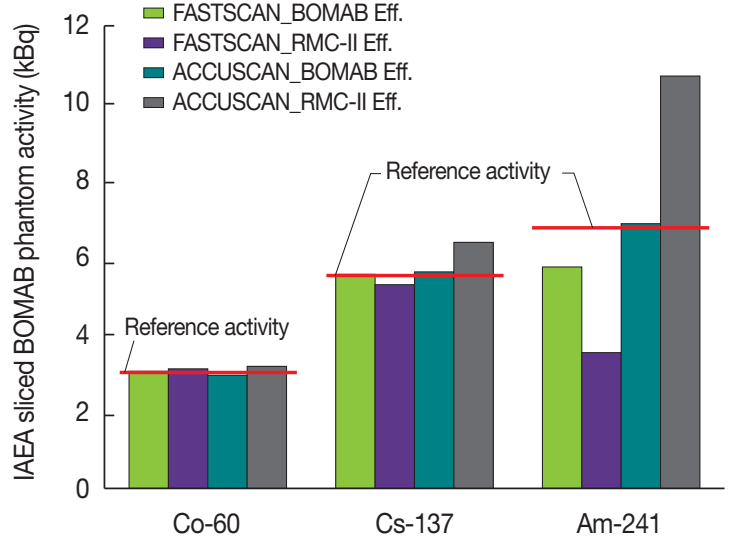

Fig. 6. Measurements made using the International Atomic Energy Agency sliced bottle manikin absorption (BOMAB) Phantom in the Fastscan and Accuscan.

\section{Results of intercomparison analysis using sliced BOMAB phantoms}

The measurements for the sliced BOMAB phantom are illustrated in Figure 6. The bars filled with diagonal lines denote values derived from the Fastscan, whereas those filled with horizontal lines denote values derived from the Accuscan. The data are also grouped to indicate results obtained using counting efficiency calibrated using the BOMAB phantom versus results obtained using the RMC-II phantom, and the reference activity of each nuclide is indicated with a horizontal line. The nuclides contained in the IAEA sliced BOM$\mathrm{AB}$ phantom are ${ }^{60} \mathrm{Co},{ }^{137} \mathrm{Cs}$, and ${ }^{241} \mathrm{Am}$. As described earlier, in our intercomparison of counting efficiency by phantom type, at photon energies exceeding $600 \mathrm{keV}$, such as for ${ }^{60} \mathrm{Co}$ $(1,172 \mathrm{keV} / 1,332 \mathrm{keV})$ and ${ }^{137} \mathrm{Cs}(662 \mathrm{keV})$, we found that the measurements were similar between the phantoms, as indicated by minimal differences in their counting efficiencies. When we evaluated the difference in the measurements using counting efficiency of the BOMAB phantom and the RMC-II phantom in the 2 types of counters, the difference in measurements of ${ }^{60} \mathrm{Co}$, which has the highest photon energy, was only $3 \%$ for the Fastscan and $7 \%$ for the Accuscan. The difference in measurements of ${ }^{137} \mathrm{Cs}$ was $-5 \%$ for the Fastscan and $15 \%$ for the Accuscan; these differences were larger than were found for ${ }^{60} \mathrm{Co}$ but still about $\pm 10 \%$. However, for nuclides with photon energies lower than $600 \mathrm{keV}$, such as ${ }^{241} \mathrm{Am}$ (59 keV), we found that the difference in measurements was $-38 \%$ for the Fastscan counter and $108 \%$ for the Accuscan counter, which is a much larger difference.

The radioactivity of the sliced BOMAB phantom, which 


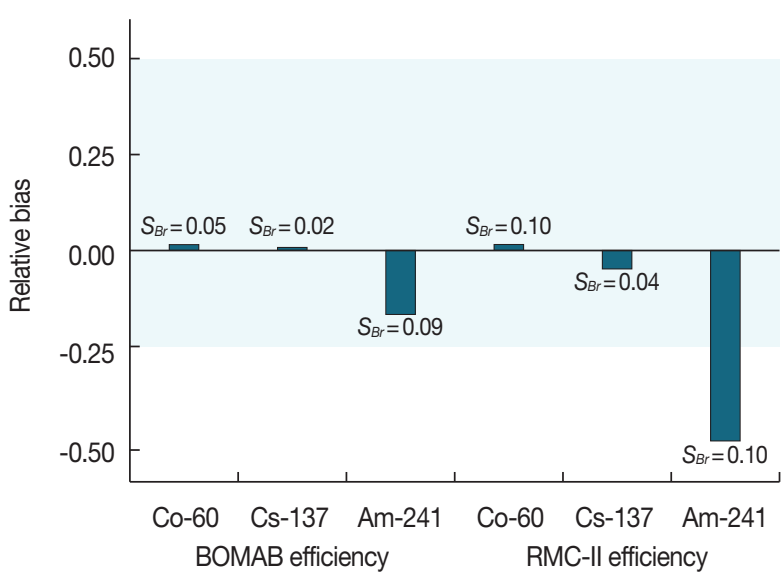

Fig. 7. Intercomparison results of bottle manikin absorption (BOMAB) phantom efficiency and Radiation Management Corporation (RMC-II) phantom efficiency in the Fastscan.

was analyzed through either the counting efficiency of the BOMAB phantom or that of the RMC-II phantom, was assessed following the ISO 28218 criteria. The results of the intercomparative analysis are presented in Figure 7 for Fastscan and in Figure 8 for Accuscan.

The $S_{B r}$ of the radioactivity measurements of the Fastscan ranged from 0.02 to 0.10 , and all measurements satisfy the acceptable performance criteria irrespective of the type of calibration phantom used. For the Fastscan, the $B_{r}$ of measurements derived from the counting efficiency of the BOM$\mathrm{AB}$ phantom ranged from -0.16 to 0.01 , satisfying the performance criteria, but those of measurements derived from the counting efficiency of the RMC-II phantom were 0.01 for ${ }^{60} \mathrm{Co},-0.05$ for ${ }^{137} \mathrm{Cs}$, and -0.49 for ${ }^{241} \mathrm{Am}$. Therefore, the $B_{r}$ values of ${ }^{241} \mathrm{Am}$ measurements obtained from the Fastscan calibrated with the RMC-II phantom were suboptimal and failed to meet the performance criteria.

Similarly to the values obtained from the Fastscan, the $S_{B r}$ values of the Accuscan (0.02-0.34) satisfied the performance criteria, irrespective of the calibration phantom used. However, whether the $B_{r}$ of the Accuscan was satisfactory depended on the calibration phantom. The $B_{r}$ of the nuclides ranged from -0.01 to and 0.03 when the BOMAB phantom counting efficiency was used, satisfying the performance criteria. However, the $B_{r}$ was 0.03 for ${ }^{60} \mathrm{Co}, 0.15$ for ${ }^{137} \mathrm{Cs}$, and 0.55 for ${ }^{241} \mathrm{Am}$ when the RMC-II phantom counting efficiency was used, which means that ${ }^{60} \mathrm{Co}$ and ${ }^{137} \mathrm{Cs}$ met the performance criteria but ${ }^{241} \mathrm{Am}$ did not.

All performance criteria were met when the efficiency calibration was performed using the BOMAB phantom, but not

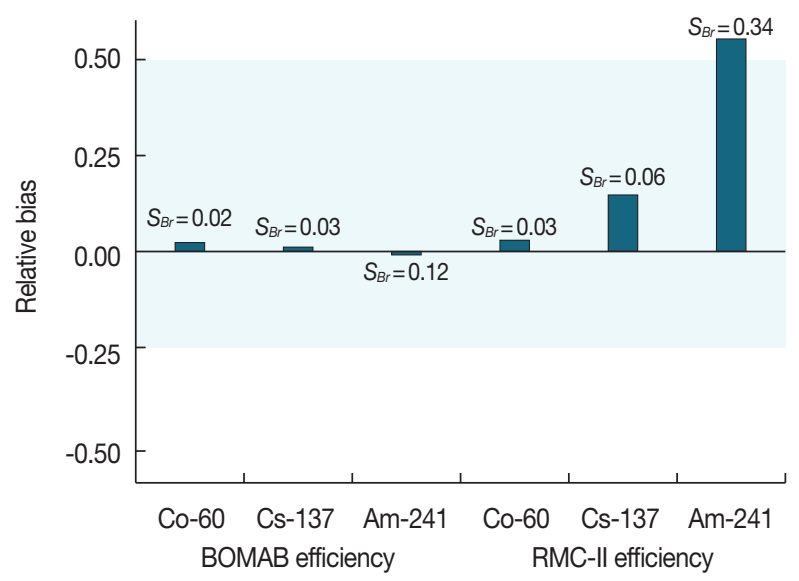

Fig. 8. Intercomparison results of bottle manikin absorption (BOMAB) phantom efficiency and Radiation Management Corporation (RMC-II) phantom efficiency in the Accuscan.

when the efficiency calibration was performed using the RMC-II phantom. The counting efficiency of the RMC-II phantom at low photon energies failed to satisfy the criteria for accurate measurements.

\section{Conclusion}

Through an intercomparative analysis between IAEA reference values and laboratory-obtained values, we compared the efficiencies of 2 whole-body counters-the Fastscan and the Accuscan-that differed in terms of detector type and geometry, after each counter was calibrated with either an adult male BOMAB phantom or an RMC-II phantom. We found that the counting efficiency of the Fastscan showed a large discrepancy between the 2 calibration phantoms for nuclides emitting energy below $600 \mathrm{keV}$ but not for nuclides emitting energy over $600 \mathrm{keV}$. Moreover, we found that the counting efficiencies of the Accuscan additionally showed a discrepancy between the 2 calibration phantoms with respect to nuclides emitting energy above $600 \mathrm{keV}$; of note, this discrepancy was more prominent than the corresponding discrepancy in the Fastscan. To investigate the effect of such disparities in counting efficiencies on the validity of measurements, we carried out an intercomparison of the 2 whole-body counters calibrated using 2 calibration phantoms. When the efficiency calibration was performed using the BOMAB phantom, we found that both whole-body counters satisfied the performance criteria. Conversely, when efficiency calibration was performed using the RMC-II phantom, we found that, for both counters, the nuclides ${ }^{60} \mathrm{Co}$ 
and ${ }^{137} \mathrm{Cs}$ met the performance standards, but the low-energy-emitting nuclide ${ }^{241} \mathrm{Am}$ did not.

For internal dosimetry laboratories, intercomparison is an effective way to ensure the quality of measurements and to improve measurement methods. Through this intercomparison, we were able to check the validation of whole-body counting systems calibrated using the BOMAB phantom. However, we found that the accuracy of the counting system calibrated using the RMC-II phantom was lower for low-energy-emitting nuclides. Our results therefore imply that efficiency calibration using the RMC-II phantom, which is potentially advantageous due to its ease of calibration and its lower cost, should be used only for high-energy-emitting nuclides. Additionally, prospective studies investigating the development of a phantom capable of faithfully reflecting the counting efficiency of the BOMAB phantom for low-energy nuclides are required.

\section{Acknowledgments}

The study was funded through the Advanced Management of National Radiation Emergency Medical Center Program (No. 50445-2016) of the Korea Institute of Radiological and Medical Sciences.

\section{References}

1. Li C, Wilkins R, Dai X, Sadi B, Ko R, Kramer GH. Canada's efforts in developing capabilities in radiological population monitoring. Health Phys. 2011;101(2):112-117.

2. International Atomic Energy Agency. Rapid monitoring of large groups of internally contaminated people following a radiation accident. IAEA-TECDOC-746. 1994;7-10.

3. Korean Agency for Technology and Standards. Quality management systems-Fundamentals and vocabulary. KS Q ISO 9000. 2007;7-11.

4. Andrasi A. whole-body counter intercomparison as a tool of quality assurance. Radiat. Prot. Dosim. 2000;89(3-4):229-233.

5. International Atomic Energy Agency. Intercalibration of in vivo counting systems using an Asian phantom. IAEA-TECDOC1334. 2003;38-40.
6. Fenwick JD, Mckenzie AL, Boddy K. Intercomparison of wholebody counters using a multinuclide calibration phantom. Phys. Med. Biol. 1991;36(2);191-198.

7. Nordic Nuclear Safety Research. In-vivo whole body measurement of internal radioactivity in the Nordic countries. ISBN 97887-7893-310-2. 2011;17-18.

8. Kramer GH, Loesch RM, Olsen PC. The second international invivo monitoring intercomparison program for whole body counting facilities by Canadian and United States agencies. Health Phys. 2001;80(3):214-224.

9. Kramer GH, Loesch RM, Olsen PC. The 1993 intercomparison of the measurement of in vivo radioactivity. Radiat. Prot. Dosim. 1999;86(3):197-205.

10. Thieme M, Hunt EL, König K, Schmitt-Hannig A, Gödde R. European whole-body counter measurement intercomparison. Health Phys. 1998;74(4):465-471.

11. American National Standards Institute/Health Physics Society. Performance Criteria for Radiobioassay. ANSI/HPS N13.30. 2011;9-10.

12. American National Standards Institute/Health Physics Society. Specifications for the Bottle Manikin Absorption Phantom. ANSI/HPS N13.35. 1999;8-13.

13. Bush F. The integral dose received from a uniformly distributed radioactive isotope. British .J Radiol. 1949;22:96-102.

14. International Commission on Radiological Protection. Report of the Task Group on Reference Man. ICRP Publication 23. 1975;826.

15. Idaho National Laboratory. Calibration of the Accuscan II In Vivo System for I-125 Thyroid Counting. INL/EXT-11-22663. 2011;97-101.

16. American National Standards Institute/Health Physics Society. Thyroid radioiodine uptake measurements using a neck phantom. ANSI N44.3. 1973;2-4.

17. The International Organization for Standardization. Radiation protection-Performance criteria for radiobioassay. ISO 28218. 2010;10-12.

18. Kramer GH, Hauck BM. The sliced BOMAB phantom: A new variant for intercomparison. Health Phys. 2006;90(2):161-166.

19. Kramer GH, Capello K, Phan Q. Effect of mass, at a fixed height, on the counting efficiency of a BOMAB phantom in three types of whole-body counter modeled by MCNP5. Health Phys. 2008; 95(2):234-240. 